\title{
Multi-Link Optical Communications System
}

\author{
Robert J. Feldmann and 1st Lt Gregory J. Toussaint
}

\author{
WL/AAAI Wright-Patterson AFB OH
}

\begin{abstract}
This in-house laboratory research effort designed, constructed, and tested a non-gimballed, omni-directional laser communications receiver system. Since laser communications depends on narrow beams of light to transmit energy and maintain covertness, it is usually difficult to initially acquire and continually track a communications signal. The optical system we are developing will help solve the problem of acquiring and tracking laser communications signals without using mechanical gimbals by providing a staring optical device with a very wide field-of-view. The system is based on a distributed array of optical receive and transmit elements, with each one responsible for a designated field-of-view.
\end{abstract}

The developed brassboard receiver array consists of small lenses, fiber optic cables, optical filters, and detector amplifiers. Each lens has a relatively narrow field-of-view (approximately 10 degrees), so collectively, the entire 20 lens array gives hemispherical coverage ( 180 degrees) in a horizontal plane. We connected each lens to a fiber optic cable which conducts the optical energy to a filter and a detector amplifier package. We interfaced all of the detectors to a signal processor which monitors their output and determines which elements are receiving energy. The processor computes the angle-of-arrival and tracks the incoming signals as they move across the individual fieldsof-view of the receiving elements, thus providing continuous tracking of the incoming communications signal.

This paper describes the design of the receiver array, the experimental receiver set up, the signal processing software that controls the system, and the system's performance. The paper concludes with recommendations for continued development of this approach.

\section{Introduction}

The objective of this in-house research effort was to design and construct a non-gimballed, omni-directional optical laser communications system. Since laser communications depends on narrow beams of light to transmit energy and maintain covertness, it is usually difficult to initially acquire and continually track a communications signal. The optical system we are developing will help solve the problem of acquiring and tracking laser communications signals without using mechanical steering devices by providing an optical device with a very wide field-of-view. The system is based on a distributed array of optical receive and transmit elements, with each responsible for a designated field-ofview.

This effort designed, constructed, and tested a simplified optical receiver array and signal processing software. The multi-faceted receiver is used to detect laser energy over a wide field-of-regard and in turn determine angle of arrival. The angle of arrival calculation is used to provide a point back direction to a laser transmitter array working in conjunction with the receiver. Beyond this effort, an integrated, receiver and transmitter array could be expanded to completely cover a hemispherical region in space and a communications modulator and demodulator added to test the system's end to end performance.

The developed receiver array consists of small lenses, fiber optic cables, optical filters, and detector amplifiers. Each lens has a relatively narrow field-of-view (approximately $10^{\circ}$ ), so collectively, the entire 20 lens array gives hemispherical coverage $\left(180^{\circ}\right)$ in a horizontal plane. We connected each lens to a fiber optic cable which conducts the optical energy to a filter and a detector amplifier package. We interfaced all of the detectors to a signal processor which monitors their output and determines which elements are receiving energy. The processor computes the angle of arrival and tracks the incoming signals as they move across the receiver, thus providing continuous receive communications. As the laser signal is tracked, this information is used to compute point back commands for the transmitting elements which are sequentially turned on to illuminate the desired area of regard.

\section{$\underline{\text { Receiver Design }}$}

The program started with a basic concept for the design of the receiver array. Initially, we envisioned an array of elements, with each element containing a simple lens connected to a fiber optic cable and then connected to a detector. The completed receiver array would be similar to a fly's eye in how it performs [1]. We wanted the fiber optic cable between the lens and the detector for three reasons. First, the cable would allow us to freely position the lens and point it in the proper direction. Second, the fiber optic cable would allow us to locate all of the detectors near each other to simplify the system's wiring requirements. Finally, in addition to these design constraints, fiber optics is a logical choice for the system because it may become an 
integral part of aircraft designs in the future. Smart skins and embedded avionics may employ fiber optics, which would be part of the aircraft structure and used to monitor stresses and damage to the airframe [2-5]. Similarly fiber optics designs could be used to create a distributed laser communications/warning receiver array, as a smart skins structure [6]. We have not investigated the details of incorporating a fiber optic communications system into future aircraft, but we believe the potential exists and are investigating our communications design with this goal in mind.

Lenses, fiber optic cables, optical filters, and detector amplifier units were purchased from General Fiber Optics. The parts were configured with standard SMA connections so we could easily assemble each element. We requested 20 copies of each part, so we could assemble a 20 element array. We specified that each lens would have a ten degree field of view, which would allow the 20 lenses to provide approximately $180^{\circ}$ coverage in a horizontal plane, considering that there is a one degree overlap between each element. We chose a ten degree field-of-view for each element, because we could create an array with a hemispherical field-of-view in a horizontal plane with a relatively small number of elements. By using only twenty elements, as opposed to a much larger number, we easily determined that the concept for the receiver array was valid. The array provided an excellent starting point for future research to find the best field-of-view for each element.

Each receive lens has a diameter of $5.0 \mathrm{~mm}$ and is housed in a small stainless steel cylinder with a diameter of $9.0 \mathrm{~mm}$ and a length of $23.5 \mathrm{~mm}$. We purchased fiber optic cables that were approximately one meter long, have a fiber core diameter of $200 \mathrm{~mm}$, and are coated in a reinforced plastic sheath. The filter cells contain an optical filter with a peak transmission at $830 \mathrm{~nm}$ and a passband of approximately $20 \mathrm{~nm}$ at half power. We chose the peak transmission wavelength based on the available laser transmitters that could be used to test the receiver. We purchased a detector amplifier unit with a peak response at $850 \mathrm{~nm}$. This was used with a simple circuit to operate the detectors. Figure 1 shows a sketch of the components in each receive element.

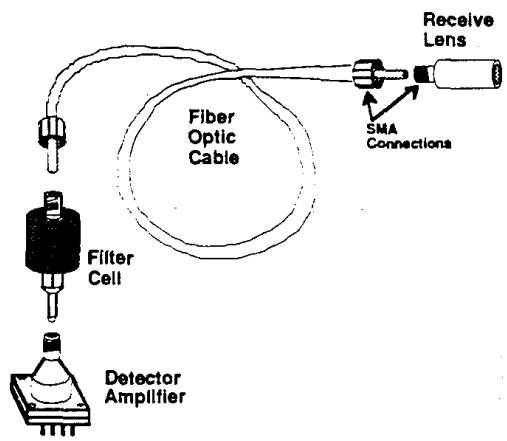

Figure 1. Sketch of receive element components.

\section{Transmitter Design}

To test our receiver, we required laser sources that could simulate communications signals. After reviewing a variety of vendors and their products, we purchased three GALA Laser Systems from D.O. Industries, Inc., Rochester, NY. Each laser transmitter consisted of a laser driver and a focusable laser collimator. The laser collimator contained a laser diode that operated at $830 \mathrm{~nm}$ and could output a continuous wave $(\mathrm{CW})$ peak power of $25 \mathrm{~mW}$. We chose the laser diode at $830 \mathrm{~nm}$ because it was readily available and had large peak power. Our choice for the transmit wavelength determined the filter wavelength for the receive elements. The focusable laser collimator allows us to easily change the beam divergence from a collimated beam to a beam with a ten degree divergence (full angle). We can also modulate the output from the transmitters by directing an analog signal into the modulation input port on the power supply unit. We purchased three transmitters so we could test the receiver's ability to track more than one incoming signal at a time. The laser systems are easy to set up and operate and they have all of the necessary safety features required for our laboratory.

\section{Experimental Set Up of Receiver Array}

We checked all of the parts to ensure that they fit together properly and then we assembled a single receiver element. We plugged the detector amplifier into a small circuit board and connected a power supply (Tektronix PS503A) to operate the device. We monitored the output of the detector with a hand held multimeter (Hewlett Packard E2377A). Figure 2 shows a wiring connections for the detector amplifier unit.

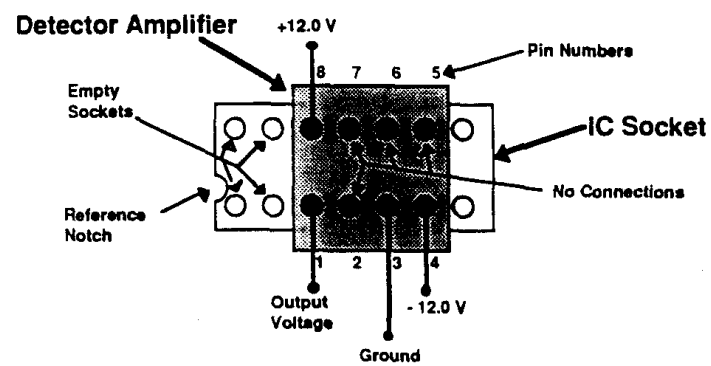

Figure 2. Pin connections for detector amplifier unit.

We mounted a receive element lens assembly into an adjustable chuck and attached it to two rotation stages to allow us to easily align the element. The two stages rotate the receive element about the horizontal and vertical axes as shown in Figure 3. 


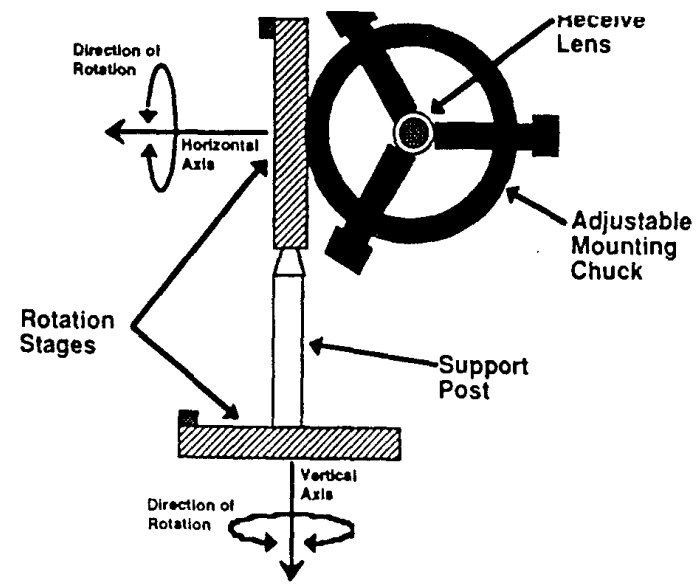

Figure 3. Receive lens mounted in adjustable chuck with rotation stages and support post.

We set up a laser transmitter and mounted it and the receiver element on to one of the smaller optical breadboards so we could measure the actual field-of-view for an individual lens. The receiver and transmit elements were $27 \mathrm{~cm}$ apart and both could be rotated to align their optical axes. We set the laser transmitter to a constant beam divergence and output power and then adjusted both elements to obtain the maximum signal at the detector amplifier. We measured both the horizontal and vertical components of the field-of-view for the receive element by rotating it about each axis independently, without moving the transmitter.

As specified by the manufacturer, the receive element has approximately a ten degree field-of-view. The horizontal and vertical components of the field-of-view are nearly identical in size. The data show that the receive element's sensitivity to the input signal decreased rapidly as the element pointed away from the optical axis of the transmitter. As we rotated the element, it presented a smaller effective lens area with which to collect the optical energy, therefore, the response decreased. In addition, we connected the lens element to the rotation stages, so that when we rotated one stage the lens moved closer or further away from the transmitter, which increased or decreased its response to the incident energy. In this case, the element's response changed because the distance between the transmitter and receiver changed and the transmit beam's energy per unit area varies inversely with distance. This information indicates that close attention must be given to each element's alignment in the receiver array to make sure there are no gaps in the coverage. A small pointing error could result in a blindspot in the receiver.

After verifying the operation of one receive element, we proceeded to construct the entire receiver array. We mounted the 20 detector amplifier units on to a large circuit board and made all of the appropriate wire connections to provide power to each element. The filter cells were attached directly to the detector amplifiers. We connected a 21 wire ribbon cable to the large circuit board with one wire for each detector output signal and the remaining wire for the circuit ground. We placed the 20 receive lenses in the adjustable chucks and attached two rotation stages to each one. We then mounted the elements on to the large optical breadboard ( $2 \mathrm{ft}$ by $3 \mathrm{ft}$ ) and connected the fiber optic cables between the lenses and the filter cells. Figure 4 shows how the receive elements were arranged on the breadboard and the direction of each element's field-ofview.

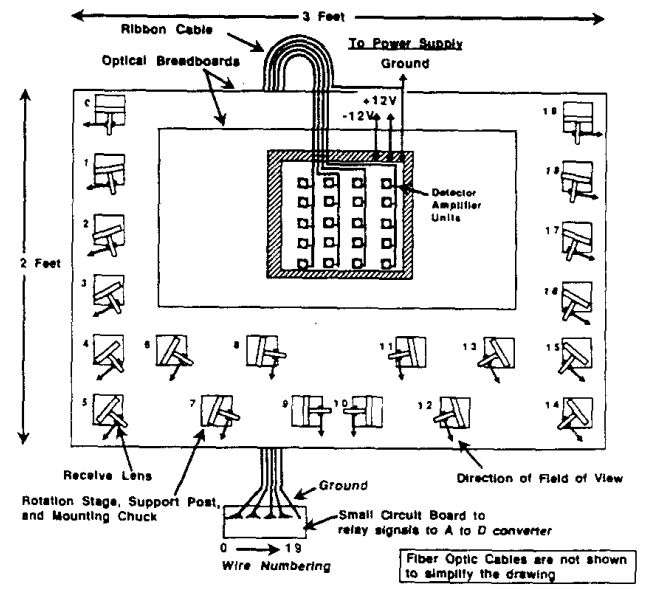

Figure 4. Optical breadboard layout for receiver array.

We directed the ribbon cable connected to the large circuit board underneath the breadboard and connected it to a smaller circuit board in front of the receiver array. The small circuit board allowed us to easily monitor the voltage at any receive element without disturbing the individual detectors on the large circuit board. We also used the small circuit board as a relay point for the detector output voltages to be connected to the analog to digital converter.

We connected a second 21 wire ribbon cable to the small circuit board and ran the wire to the analog to digital (A to D) converter, which is connected to Digital MicroVAX computer. The $\mathrm{A}$ to $\mathrm{D}$ converter, Model number $\mathrm{ADQ32}$, is capable of accepting up to 32 input signals at one time. We connected our detector outputs to the first 20 channels on the converter and we connected the circuit ground to the ground terminal. The $\mathrm{A}$ to $\mathrm{D}$ converter can sample signals at rates up to $200 \mathrm{kHz}(200,000$ samples per second), but the exact sampling rate depends on how the converter is configured by the computer. We developed a computer program in the $C$ language to control the $A$ to $D$ converter and monitor the detector voltage levels.

\section{Signal Processing Software}

We wrote the signal processing software in the $\mathrm{C}$ language which is an efficient language for controlling the $A$ to $D$ converter. The program presents the user with a menu of four options for monitoring the output voltages from the detectors. 
First, the user can view a chart of the raw detector voltages and the normalized voltages. The chart can be updated as many times as the user wants. The raw voltages are the actual values output by the detectors and the normalized voltages are the raw voltages multiplied by a factor which makes each detector have the same normalized voltage for a given amount of input optical energy. The detectors are all slightly different, so the normalization factors help correct the differences and make it possible to directly compare the output from two detectors.

The second and third options for monitoring the detectors are both graphs. The user can view either a bar graph or a line graph of the normalized voltages. The user can repeatedly update each graph or choose to return to the main menu.

The final program option is to monitor only the detectors that are receiving a communications signal and to track the signals as they move across the receiver. The program compares each normalized voltage to a threshold value to determine if a signal is present. If the program detects a signal, then it labels the signal and keeps the same label attached to that signal if it moves continuously around the receiver. If a signal disappears and later returns or if it jumps from one receive element to a non-adjacent element, then the software considers it a new signal and gives it a new label. We designed the program to track up to three signals and display a warning message if more than three signals are present. The software can easily be modified to track more signals if needed.

\section{System Performance}

The receiver array and signal processing software perform the acquisition and tracking functions extremely well. We set the signal threshold level at $10 \mathrm{mV}$ of normalized voltage to reduce the number of false alarms generated by noise in the system. At this threshold level, the processor can still detect actual signals since the typical signal level is above 20 $\mathrm{mV}$. We verified that all four display options work correctly and we made final adjustments with the receiver array to make sure it provided continuous coverage over $180^{\circ}$ in a horizontal plane. We evaluated the chart and the two graphing display options by pointing transmit signals at the array and watching the output on the computer screen. We monitored the voltage at the detector with a hand held multimeter and compared the values to the signal processor's outputs. We found no problems with the chart and graphing options. The tracking option required more detailed testing to make sure it was applying labels correctly.

We tested the tracking display option by pointing different sequences of one, two, and three transmit lasers at the receiver array. With one transmit laser, the signal processor assigned a label to it and maintained that label as long as the signal moved continuously around the receiver. Since there was only one signal, even if it moved discontinuously or if it disappeared and then reappeared, it maintained the same label. The processor handled two signals equally well as they moved around the receiver and if one signal moved discontinuously it again retained the same label, because there were only two signals. To fully test the labeling function, we turned on three transmit lasers. The processor accurately maintained labels as the signals moved and correctly assigned labels as signals disappeared and reappeared. For example, we turned on all three transmitters to create three signals and then blocked the transmitters corresponding to signal labels two and three. We then removed the block from the transmitter that had been signal label three and when it reappeared it was correctly labeled as signal number two. This example shows that the processor can keep track of the current labeled signals and correctly label new signals when they appear. We performed several other tests to verify the labeling function's performance as part of the tracking display option and found no errors.

\section{Summary}

We successfully demonstrated a wide field of view optical receiver in the laboratory. The receiver is a distributed array of 20 individual elements, each responsible for a $10^{\circ}$ field of view. The combined elements provide $180^{\circ}$ coverage in a horizontal plane. We interfaced the receive elements through an analog to digital converter to a Digital MicroVAX computer, which served as a host for the signal processing software for the system. The signal processor can monitor all 20 receive elements and can track up to three independent communications signals as they move around the receiver. We calculated normalization factors for the receiver so that each element would have the same response to a given amount of input optical energy. Point back angles were computed for a transmitter which could then close the communications link.

\section{References}

1. Horridge, G.A. "The Compound Eye of Insects," Scientific American, Vol 237, 1, July 1977, 108-120.

2. Udd, E. "Fiber Optic Smart Structures and Skins," SPIE Proceedings, Vol 986, (1988), 2-5

3. Vengsarkar, A. M. and Murphy, K. A. " Fiber Sensors in Aerospace Applications: The Smart Structures Concept," Photonics Spectra, April 1990, 119-124.

4. Claus, R. O. "Fiber Sensors as Nerves for 'Smart Materials'," Photonics Spectra, April 1991, 85-88.

5. Talat, $\mathrm{K}$ "Fiber Sensors Take Wing in Smart-Skin Applications," Photonics Spectra, April 1991, 85-88.

6. Morrison, D. C. "Military Optronics: From 'Fly-byLight' to 'Smart Skins'," Lasers and Optronics, September 1989, 28-30. 